ARTICLE

https://doi.org/10.1038/s41467-019-08386-9

\title{
Enabling low voltage losses and high photocurrent in fullerene-free organic photovoltaics
}

Jun Yuan 1,2,3, Tianyi Huang ${ }^{1}$, Pei Cheng (1) ${ }^{1}$, Yingping Zou ${ }^{2}$, Huotian Zhang (D) ${ }^{4}$, Jonathan Lee Yang ${ }^{5}$, Sheng-Yung Chang ${ }^{1}$, Zhenzhen Zhang ${ }^{2}$, Wenchao Huang ${ }^{1}$, Rui Wang ${ }^{1}$, Dong Meng ${ }^{1,3}$, Feng Gao id ${ }^{4}$ \& Yang Yang ${ }^{1,3}$

Despite significant development recently, improving the power conversion efficiency of organic photovoltaics (OPVs) is still an ongoing challenge to overcome. One of the prerequisites to achieving this goal is to enable efficient charge separation and small voltage losses at the same time. In this work, a facile synthetic strategy is reported, where optoelectronic properties are delicately tuned by the introduction of electron-deficient-core-based fused structure into non-fullerene acceptors. Both devices exhibited a low voltage loss of $0.57 \mathrm{~V}$ and high short-circuit current density of $22.0 \mathrm{~mA} \mathrm{~cm}^{-2}$, resulting in high power conversion efficiencies of over $13.4 \%$. These unconventional electron-deficient-core-based non-fullerene acceptors with near-infrared absorption lead to low non-radiative recombination losses in the resulting organic photovoltaics, contributing to a certified high power conversion efficiency of $12.6 \%$.

\footnotetext{
${ }^{1}$ Department of Materials Science and Engineering, University of California, Los Angeles, Los Angeles, CA 90095, USA. ${ }^{2}$ College of Chemistry and Chemical Engineering, Central South University, 410083 Changsha, China. ${ }^{3}$ California NanoSystems Institute, University of California, Los Angeles, Los Angeles, CA 90095, USA. ${ }^{4}$ Department of Physics, Chemistry and Biology (IFM), Linköping University, 58183 Linköping, Sweden. ${ }^{5}$ College of Chemistry, University of California, Berkeley, CA 94720, USA. These authors contributed equally: Jun Yuan, Tianyi Huang, Pei Cheng Correspondence and requests for materials should be addressed to Y.Z. (email: yingpingzou@csu.edu.cn) or to F.G. (email: feng.gao@liu.se) or to Y.Y. (email: yangy@ucla.edu)
} 
$\mathrm{O}$ rganic photovoltaics (OPVs) have several advantages such as potential low cost, absorption tunability, mechanical flexibility, and roll-to-roll manufacture capability $^{1-11}$. However, the power conversion efficiencies (PCEs) of OPVs are still inferior to their inorganic counterparts. One of the most significant factors, which hinders their device performance, is the relatively large loss in the open-circuit voltage $\left(V_{\mathrm{OC}}\right)$ with respect to the optical gap ${ }^{12}$. While the $V_{\text {OC }}$ loss can be as low as $0.30 \mathrm{eV}$ in $\mathrm{GaAs}$ and slightly higher in $\mathrm{c}-\mathrm{Si}$ and organic-inorganic hybrid perovskite solar cells (from 0.40 to $0.55 \mathrm{eV}$ ), the $V_{\mathrm{OC}}$ loss for strongly absorbed photons in state-of-the-art OPVs is around $0.6 \mathrm{~V}$ or higher ${ }^{13-15}$. In an ideal OPV, maximum $V_{\mathrm{OC}}$ can be achieved only when sources of voltage losses are limited to an unavoidable radiative recombination of absorption species above the optical gap ${ }^{16}$. However, for the state-of-the-art OPVs, there are strong nonradiative recombination present leading to significant voltage losses, evidenced by electroluminescence quantum efficiency $\left(\mathrm{EQE}_{\mathrm{EL}}\right)$ measurements ${ }^{17-19}$. Recombination of radiative charge-transfer (CT) states can also be present due to energetic offsets between the donor (D) and the acceptor (A) components $^{20-22}$. In a very recent work ${ }^{23}$, two design rules are formulated aiming at reducing the voltage loss and increasing the efficiency of OPVs: (1) Small energy offset between the donor and acceptor materials, and (2) high luminescence yield of the lowergap single component (and hence the blend). Among the materials candidates meeting these design rules, A-D-A-structured non-fullerene acceptors (NFAs) are extremely promising since they exhibit strong intramolecular charge-transfer (ICT) effect, superior energy level tunability, and good molecular crystallinity ${ }^{24-27}$. These features allow NFAs to become potential candidates for achieving an efficient charge separation and low voltage losses simultaneously as per the design rules.

From the aspect of molecular design, synthesis of donor and acceptor materials with complementary absorption profiles intended to maximize the coverage of the solar spectrum has been one of the prerequisites for achieving a high photocurrent. Thus, a variety of narrow optical gap NFAs based on a stronger electrondonating at the central core were carefully designed and synthesized for OPVs ${ }^{28}$. Undoubtedly, it is a successful strategy to elevate the HOMO level reducing the optical gap. Benzotriazole-based conjugated molecules with unique luminescence properties are common building blocks for increasing the photoluminescence quantum yield (PLQY) of the single component ${ }^{29-32}$. The high PLQY indicates efficient radiative recombination pathways, which may result in high electroluminescence yield of the resulting devices. Hence, it attempts to introduce benzotriazole into the central core to form an electron-deficient-core-based fused structure $(\mathrm{DAD})$ for adjusting the optoelectronic properties of the resulting molecules, aiming for a small voltage loss and high performance in devices. By using DAD fused core one could also adopt a relatively planar structure rather than a twisted one and hence, facilitate the electron transfer from the donor to acceptor since the planarized DAD enhances the delocalization of $\pi$ electrons. More importantly, a low perturbation of electrons may occur in DAD fused core, which will result in efficient charge transfer ${ }^{33}$. In other words, $\pi$-orbital electrons are less likely to be trapped while passing through the fused $\pi$-bridge upon introduction of the triazole in this fused system ${ }^{34}$.

In this work, we report a certified high efficiency of singlejunction OPVs based on two fused-ring NFA molecules (Y1 and Y2) consisting of dithienothiophen[3,2-b]pyrrolobenzotriazole (BZPT) group with non-halogenated dicyanomethylene derivatives (INIC or INTC). A simple design strategy of covalent nitrogen bridge with adjacent thieno[3,2-b]thiophene and benzotriazole makes it possible to delicately tune the optoelectronic properties of target molecules, resulting in an improvement of optical coverage of near-infrared region (NIR) spectrum. A commercial donor polymer PBDB- $\mathrm{T}^{35}$ is selected because of its negligible band offsets with Y1 and Y2. In spite of this small energetic offset, which results in relatively high $\mathrm{EQE}_{\mathrm{EL}}$ (approximately $0.5 \times 10^{-4}$ ) and small voltage losses, charge separation is still efficient and offers decent short-circuit current density (over $22 \mathrm{~mA} \mathrm{~cm}^{-2}$ ), resulting in PCEs of over $13.4 \%$ (13.42\% for Y1 and $13.40 \%$ for Y2). These devices have been certified at the photovoltaic Lab of Newport Corporation, showing a $12.6 \%$ efficiency, which is the high efficiency of current single-junction organic cells meeting the ISO 17025 Standards reported so far. This remarkable feature of the presented fused benzotrizole-based system serves as an inspiration to design nextgeneration NFAs for high-performance OPVs.

\section{Results}

Characterization of non-fullerene acceptors. To meet the rules mentioned above, our molecule design rational consists of tuning the HOMO levels relatively close to that of the donor and, meanwhile, lowering the LUMO levels to make the absorption spectra of donor material and acceptor materials complementary:

(1) Introducing the nitrogen atoms in the center core unit serve as heteroatomic bridges for covalent planarization ${ }^{36}$ : this provides stronger electron-donating character and allow for charge carrier mobility in contrast to cyclopentadiene (the center structural unit of the IDTT) ${ }^{37,38}$, thus increasing the HOMO energy levels;

(2) Adding a weak electron-withdrawing moiety of 2-ethylhexyl-benzo[d]- $[1,2,3]$-triazoles segment ${ }^{39}$ in the middle of the central core to form a fused DAD structure: this helps to improve the efficient radiative recombination pathway and enhance the electroluminescence yield of the single components. Based on such a rationale, we are expecting a minimized voltage loss, which enables a high open-circuit voltage and high short-circuit current at the same time.

From the materials design point of view, in contrast to previous works ${ }^{39}$, we increase the central fused rings from pentacyclic to heptacyclic, and successfully tune the HOMO energy level to match a small band offset with the PBDB-T, meeting the requirement of the first design rule. In addition, we introduce benzotriazole unit in central core to increase the electroluminescence yield of both the single component, meeting the requirement of the second design rule. From the materials synthesis point of view, the central core that has to be sterically hindered to prevent over aggregation ${ }^{19}$ while maintaining an intramolecular charge transport channel is assumed by the alkyl side chains onto the nitrogen atoms ${ }^{40}$. In contrast to previous designs, it does not need to synthesize spiro-like structures, and this is a good example of non-spiro-like molecule that show excellent performance as an NFA. The chemical structures of Y1 and Y2 are depicted in Fig. 1a, and the detailed synthesis processes are described in Supplementary Figure 1. Nuclear magnetic resonance spectra and high-resolution mass spectrum (MALDI-TOF) of the intermediates, Y1 and Y2 are shown in Supplementary Figs 2-8. Both, Y1 and Y2, can be easily solubilized in common organic solvents, such as chloroform and chlorobenzene at room temperature. Thermogravimetric analysis curves indicate that $\mathrm{Y} 1$ and $\mathrm{Y} 2$ exhibit excellent thermal stability with decomposition temperatures at $350{ }^{\circ} \mathrm{C}$ (Supplementary Fig. 10).

Figure $1 \mathrm{~b}$ shows the normalized absorption spectra of Y1 and Y2 in solution (chloroform) and in thin films (corresponding optical data in Supplementary Table 1). For the thin films, the absorption peak red-shifts from 738 to $802 \mathrm{~nm}$ for Y1 and 758 to 
a
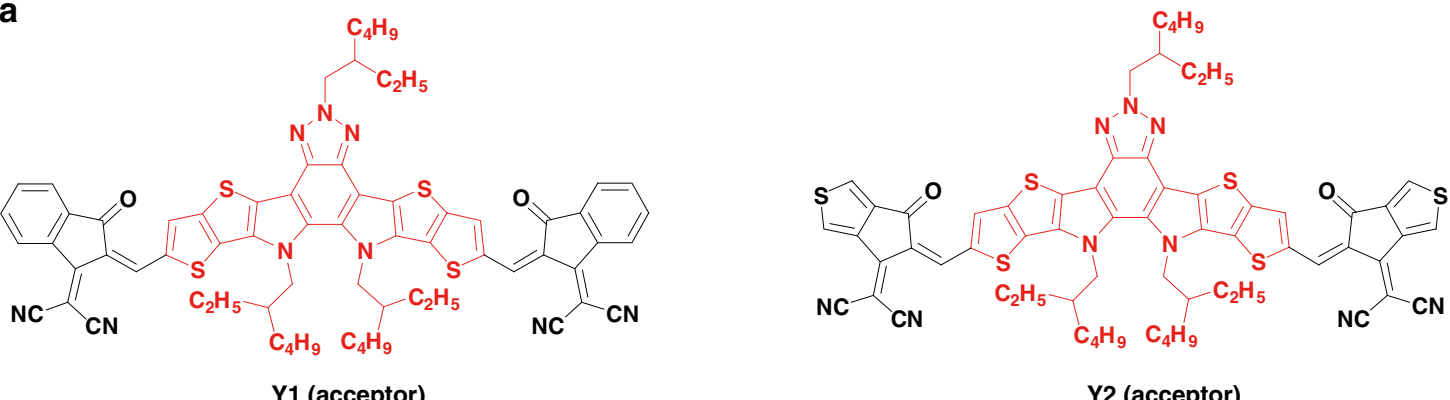

Y1 (acceptor)

Y2 (acceptor)
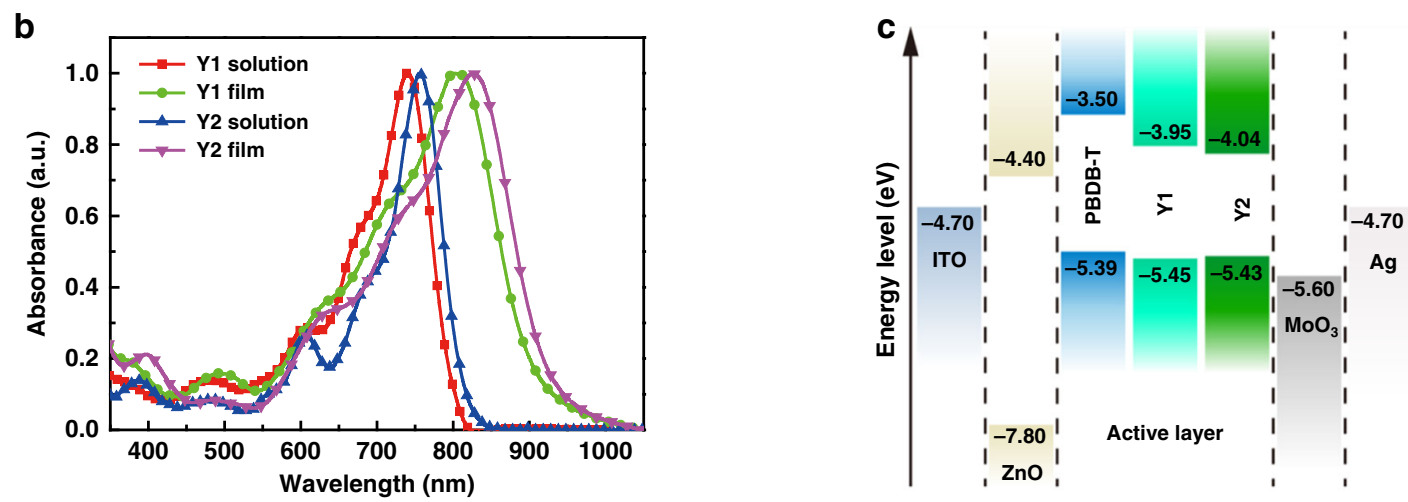

Fig. 1 Molecular structure and properties. a Chemical structure of the acceptor molecules. b Normalized absorption spectra of the acceptors Y1 and Y2. c Energy diagrams of the materials used in OPVs. OPV organic photovoltaic

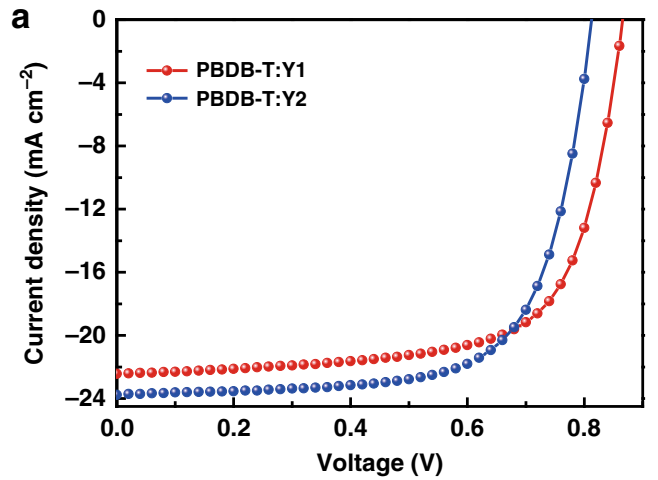

b

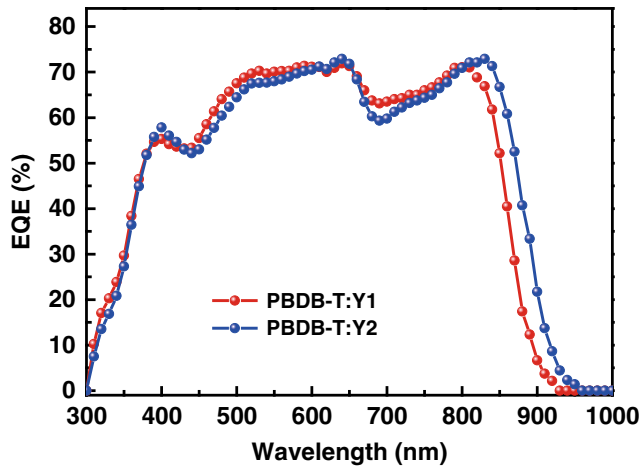

C
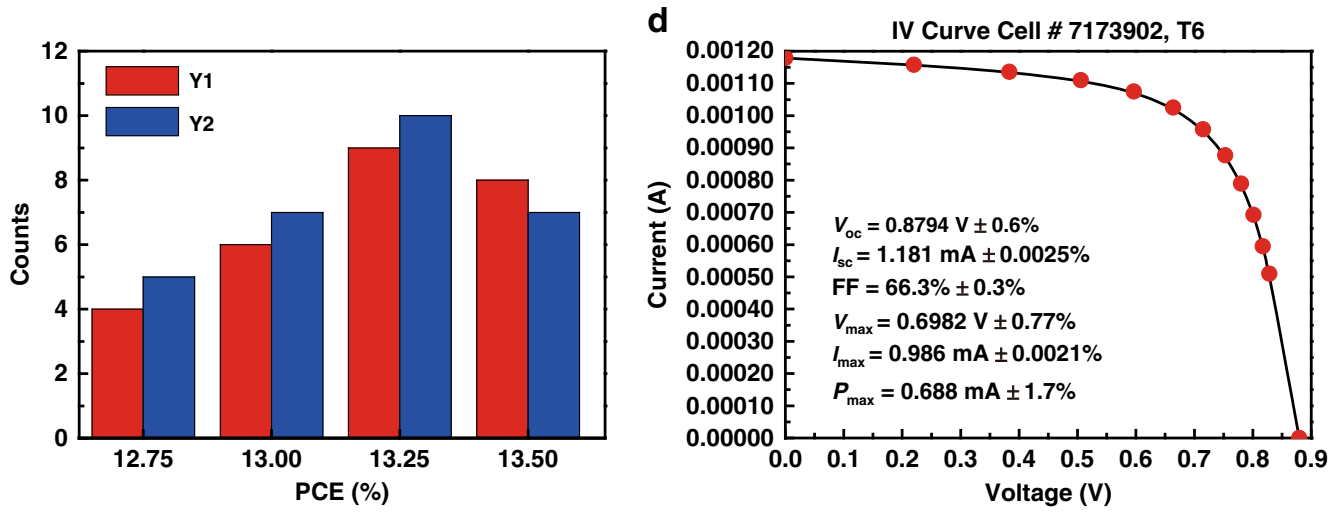

Fig. 2 Photovoltaic performance. a The $J-V$ curves of PBDB-T:Y1 and :Y2 blend solar cells. b The EQE curves of PBDB-T:Y1 and :Y2 blend solar cells. c Statistical diagram of efficiency of PBDB-T:Y1 and :Y2. d Current-voltage parameters of PBDB-T:Y1 device certified $\left(0.0548 \mathrm{~cm}^{2}\right.$ device area) by Newport Corp. EQE electroluminescence quantum efficiency 
Table 1 Photovoltaic performances of PBDB-T:Y1- and PBDB-T:Y2-based OPV devices

\begin{tabular}{|c|c|c|c|c|}
\hline PBDB-T:Y2 & $0.82(0.81 \pm 0.01)$ & $23.56(23.12 \pm 0.78)$ & $69.4(70.80 \pm 0.70)$ & $13.40(13.25 \pm 0.39)$ \\
\hline
\end{tabular}

Table $2 V_{\text {oc }}$ loss profile of PBDB-T:Y1- and PBDB-T:Y2-based OPV devices

\begin{tabular}{|c|c|c|c|c|c|c|c|}
\hline Devices & $E_{g}(e V)$ & $q V_{\text {oc }}^{\mathrm{SQ}}(\mathrm{eV})$ & $q V_{\mathrm{OC}}^{\mathrm{rad}}(\mathrm{eV})$ & $\Delta E(\mathrm{eV})$ & $\Delta E_{1} E_{\text {gap }}-q V_{\text {oc }}^{S Q}(e V)$ & $\Delta E_{2} q \Delta V_{\text {oC }}^{\text {rad,below gap }}(\mathrm{eV})$ & $\Delta E_{3} q \Delta V_{\text {oC }}^{\text {non-rad }}(\mathrm{eV})$ \\
\hline PBDB-T:Y2 & 1.40 & 1.13 & 1.09 & 0.57 & 0.27 & 0.04 & 0.26 \\
\hline
\end{tabular}

$827 \mathrm{~nm}$ for Y2. The optical gap of pure Y2 film estimated from the intersection between absorption and emission is about 1.40 $\mathrm{eV}$, which is lower than that of the pure Y1 $\left(E_{\mathrm{g}}=1.44 \mathrm{eV}\right.$, Supplementary Fig. 11). Cyclic voltammetry (CV) measurements (Supplementary Fig. 12) were employed to evaluate the electrochemical properties, showing similar HOMO levels of $-5.45 \mathrm{eV}$ for $\mathrm{Y} 1$ and $-5.43 \mathrm{eV}$ for $\mathrm{Y} 2$ due to the identical core center, and a lower LUMO level for Y2 $(-4.04 \mathrm{eV}$ compared with $-3.95 \mathrm{eV}$ for Y1) due to the stronger electron-withdrawing character of INTC compared with that of INIC (Supplementary Fig. 1). As shown in Fig. 1c, although the $\triangle \mathrm{HOMO}$ offset between the PBDB-T donor (Supplementary Fig. 9a) and Y1 or Y2 appears to be small $(0.07$ $\mathrm{eV}$ for PBDB-T:Y1 and $0.05 \mathrm{eV}$ for PBDB-T:Y2), efficient charge separation can still be achieved at the bulk heterojunction (BHJ) interface, meanwhile, with suppressed voltage losses. This will be discussed in detail in voltage losses section.

Device performance of OPVs. OPVs were fabricated with an inverted device structure of $\mathrm{ITO} / \mathrm{ZnO} /$ active layer $/ \mathrm{MoO}_{3} / \mathrm{Ag}$ (Supplementary Fig. 9b), where PBDB-T was chosen as the donor material. The donor/acceptor ratios in the active layer were carefully optimized (Supplementary Table 2). Figure 2a shows the current density versus voltage $(J-V)$ characteristics under the illumination of AM $1.5 \mathrm{G}, 100 \mathrm{~mW} \mathrm{~cm}^{-2}$. Table 1 collects the photovoltaic parameters of these OPV devices based on Y1 and Y2, respectively. Meanwhile, the statistical diagram of efficiency of PBDB-T:Y1 and :Y2 is shown in Fig. 2c. Both BZPT-based NFAs, Y1 and Y2, exhibited $J_{\mathrm{SC}}$ over $22.0 \mathrm{~mA} \mathrm{~cm}^{-2}$ and resulted in PCEs of over $13 \%(13.42 \%$ for $\mathrm{Y} 1$ and $13.40 \%$ for $\mathrm{Y} 2$, respectively). In Fig. 2b, the external quantum efficiency (EQE) spectra for Y1-based and Y2-based devices demonstrated similar shapes, while the Y2-based device displayed a $25 \mathrm{~nm}$ red-shift photocurrent response compared with that of $\mathrm{Y} 1$, which was in agreement with the trend of their absorption spectra. For devices with best $I-V$ performance, the integrated $J_{\mathrm{SC}}$ values from EQE spectra with AM $1.5 \mathrm{G}$ reference spectrum of PBDB-T:Y1 and PBDB-T:Y2 were calculated to be 22.14 and $23.18 \mathrm{~mA} \mathrm{~cm}^{-2}$, respectively, consistent with the $J_{\mathrm{SC}}$ values obtained from the $J-V$ curves. Although some previous reports achieved similarly low voltage losses, none of them could keep 60-70\% EQE from almost $400 \mathrm{~nm}$ to $860 \mathrm{~nm}^{41,42}$, which result in the high $J_{\mathrm{sc}}$ and high PCE of Y1-based and Y2-based devices.
In order to carefully evaluate the PCE, OPVs based on the PBDB-T:Y1 blend were sent to the PV calibration Lab of Newport Corporation, accredited by A2LA to ISO 17025 Standards, for certification measurements. With the current-voltage parameters shown in Fig. 2d and Table 1, detailed measurements can be found in the section of Methods (Fabrication and measurement of devices). An efficiency of $12.6 \%$ was certified with $J_{\mathrm{SC}}$ of 21.55 $\mathrm{mA} \mathrm{cm}^{-2}$, a $V_{\mathrm{OC}}$ of $0.88 \mathrm{~V}$ and an fill factor (FF) of $66.3 \%$ (Supplementary Fig. 14). The certification confirmed that OPV devices can indeed endure the standard measurement and achieve results relatively close to our regular testing, indicating a reliable and reproducible high performance of our systems. ${ }^{48}$

Voltage losses and charge separation. In order to evaluate the voltage loss in our system and unravel the nature of its high photovoltaic performance, we investigated voltage losses in both solar cells. The calculated results are presented in Table 2 . The total energy loss $(\Delta E)$ was defined as the difference between the optical gap of blend solar cell and the $V_{\mathrm{OC}}$. As shown in Table 2, $\Delta E$ is $0.57 \mathrm{eV}$ for both systems. Specification of the three sources of $V_{\mathrm{OC}}$ loss follows Equation 1 below:

$$
\begin{aligned}
\Delta E & =E_{\mathrm{g}}-q V_{\mathrm{OC}} \\
& =\left(E_{\mathrm{g}}-q V_{\mathrm{OC}}^{\mathrm{SQ}}\right)+\left(q V_{\mathrm{OC}}^{\mathrm{SQ}}-q V_{\mathrm{OC}}^{\mathrm{rad}}\right)+\left(q V_{\mathrm{OC}}^{\mathrm{rad}}-q V_{\mathrm{OC}}\right) \\
& =\left(E_{\mathrm{g}}-q V_{\mathrm{OC}}^{\mathrm{SQ}}\right)+q \Delta V_{\mathrm{OC}}^{\mathrm{rad}, \text { below gap }}+q \Delta V_{\mathrm{OC}}^{\mathrm{non}-\mathrm{rad}} \\
& =\Delta E_{1}+\Delta E_{2}+\Delta E_{3}
\end{aligned}
$$

The $\Delta E_{1}$ is the difference between optical gap and maximum voltage based on the Shockley-Queisser limit (SQ limit), caused by radiative recombination originating from the absorption above the optical gap, typically $0.25 \mathrm{eV}$ or above, unavoidable for any kind of solar cells. Here, $q V_{\mathrm{OC}}^{\mathrm{SQ}}$ is the maximum voltage based on the SQ limit, where the EQE is assumed to be 1 above the gap and 0 below the optical gap ${ }^{43}$ and the only loss is from the mismatch between radiation received in a narrow solid angle from the sun and omnidirectional radiative recombination. Radiativerecombination loss $\left(\Delta E_{2}\right)$ is due to absorption below the optical gap. Here, $V_{\mathrm{OC}}^{\mathrm{rad}}$ is the open-circuit voltage when there is only 

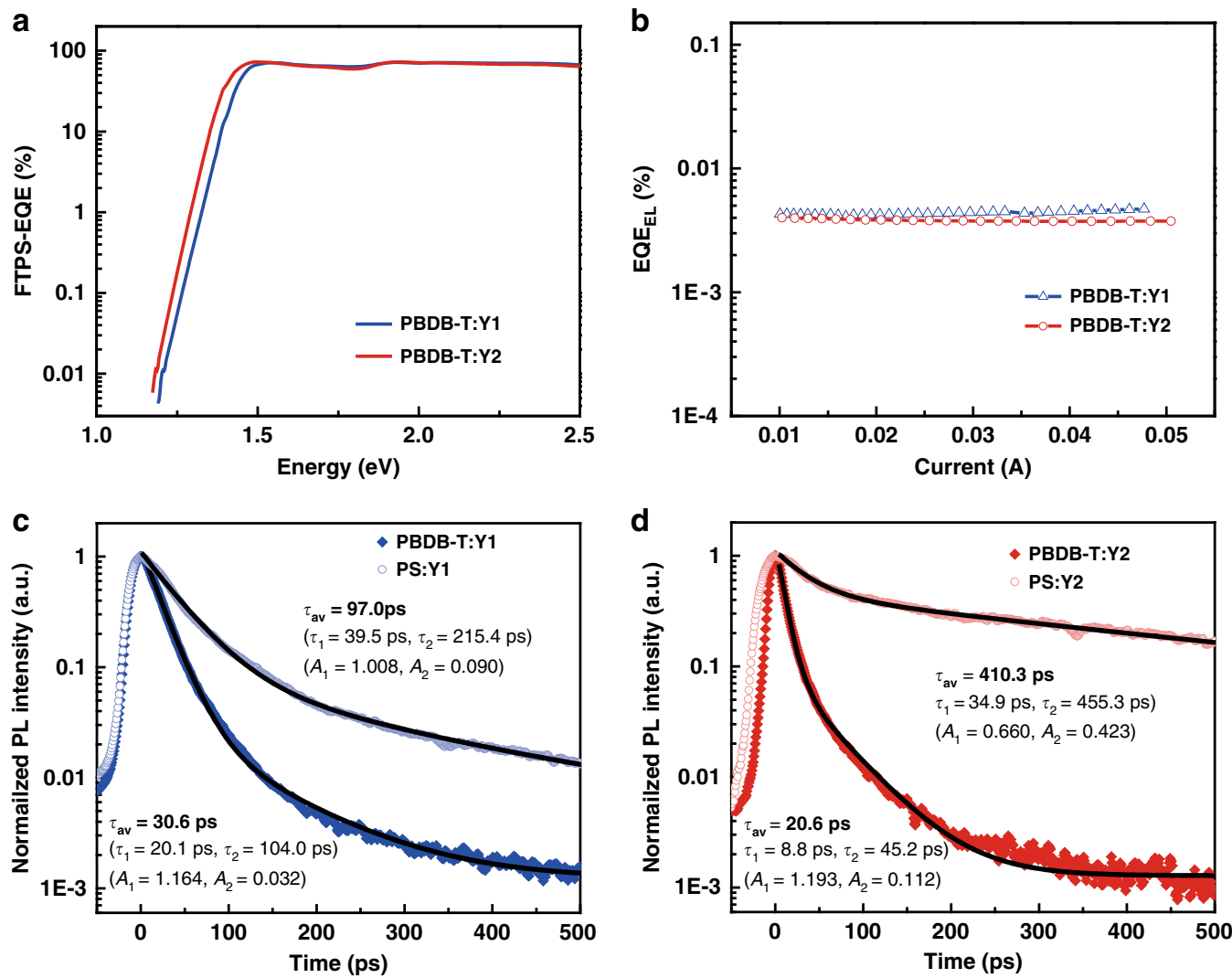

Fig. 3 Optical and electrical characterizations. a Fourier-transform photocurrent spectroscopy of PBDB-T:Y1 and :Y2 blend solar cell. b The electroluminescence quantum efficiency of PBDB-T:Y1 and PBDB-T:Y2 blend solar cells at different injected currents. c, $\mathbf{d}$ Time-resolved for D:A blends and polystyrene blends. Black solid curves are fitting curves for extracting the lifetime $\left(\tau_{\mathrm{av}}=\frac{\Sigma A_{i} \tau_{i}^{2}}{\Sigma \mathrm{A}_{i} \tau_{i}}\right)$.

radiative recombination, including both the radiative loss mentioned in $\Delta E_{1}$ and the additional radiative recombination from the absorption below the optical gap due to the nonstep function absorption. For solar cells with steep absorption edges, like inorganic or perovskite solar cells, this term is negligible. However, this part can be very high for OPVs, especially for those with obvious charge-transfer-state absorption. While in our case, the absorption onset of $\mathrm{BHJ}$ devices is sharp (Fig. 3a), leading to $q \Delta V_{\mathrm{OC}}^{\text {rad,below gap }}$ estimated as low as 0.04 and $0.05 \mathrm{eV}$, significantly smaller than that observed in typical OPVs and even most smalloffset OPVs (Supplementary Table 3). The final part of energy loss stem from the nonradiative recombination $\left(\Delta E_{3}\right)$ and could be directly obtained from the equation: $\Delta V_{\mathrm{OC}}^{\mathrm{non}-\mathrm{rad}}=\frac{k T}{q} \ln \left(\frac{1}{\mathrm{EQE}_{\mathrm{EL}}}\right)$. $\mathrm{EQE}_{\mathrm{EL}}$ results are shown in Fig. 3b. It is notable that both systems show low nonradiative losses of 0.25 and $0.26 \mathrm{eV}$ respectively, which is very low among OPVs reported with such high efficiency (Supplementary Table 3).

We also investigated the PLQY and photoluminescence (PL) quenching for the blend films. Following our previous publication, we blend the insulating polymer polystyrene (PS) with the acceptor materials, to mimic the dispersed morphology in the active layer of the devices ${ }^{23}$. The PLQY values of PS:Y1 and PS:Y2 blends are 5.8\% and 3.4\% respectively; while those of the PBDB$\mathrm{T}: \mathrm{Y} 1$ and PBDB-T:Y2 are $0.1 \%$ and $0.3 \%$, respectively, indicating efficient PL quenching. The corresponding PLQY data are summarized in Supplementary Table 4. Although efficient PL quenching indicates efficient charge separation, it also implies additional nonradiative recombination losses, which are to be further decreased. In an ideal case, the PL quenching is expected to happen at the short-circuit conditions, rather than the opencircuit conditions. Efficient PL quenching is further confirmed by the PL decay measurements (Fig. 3c, d), which show shortening of the PL lifetime in the active layers for both Y1 and Y2 blends. Consistent with our and other's previous reports ${ }^{23,44}$, the timescale on which the PL quenching occurs is very slow compared with traditional fullerene-based devices (those with large energetic offset between the donor and acceptor materials).

Film morphologies. The molecular packing characteristics of Y1 and Y2 in thin film blend with polymer PBDB-T were investigated using grazing incidence wide-angle $\mathrm{X}$-ray scattering (GIWAXS $)^{45}$. 2D GIWAXS patterns and their corresponding intensity line-profiles in out-of-plane (OOP) and in-plane (IP) directions are presented in Supplementary Fig. 15a and Fig. 16, respectively. PBDB-T:Y1 and PBDB-T:Y2 blends show similar molecular packing. In both blends, a broad peak corresponding to the $\pi-\pi$ stacking was observed along the out-of-plane direction at $q=1.7 \AA^{-1}$, which benefit efficient photon absorption and charge transport.

The morphologies were further investigated by atomic force microscopy (AFM) and transmission electron microscopy (TEM). As shown in Supplementary Fig. 15b, the formation of obvious ordered nano-fibrillar structures of active layer films were observed using AFM, accompanied with a smooth root-meansquare roughness $\left(R_{\mathrm{q}}\right)$ of 1.22 and $1.60 \mathrm{~nm}$ on a scan area of 1 $\mu \mathrm{m} \times 1 \mu \mathrm{m}$ for Y1- and Y2-based blends, respectively. The phase separation was further visualized using TEM with suitable domain sizes in both blends (Supplementary Fig. 15c), indicating an ideal phase separation for the optimized balance between 
charge transportation pathway and interface recombination. As a consequence of the orientation, both polymer and non-fullerene molecules in the active layer are observed to closely stack. The lateral charge transportation was also proven to be efficient with space charge limited current (SCLC) method ${ }^{46}$, with the average hole and electron mobilities calculated and shown in Supplementary Fig. 18. Hole mobilities of the PBDB-T:Y1 and PBDB-T: Y2 blend films were measured to be $1.56 \times 10^{-3} \mathrm{~cm}^{2} \mathrm{~V}^{-1} \mathrm{~s}^{-1}$ and $2.59 \times 10^{-3} \mathrm{~cm}^{2} \mathrm{~V}^{-1} \mathrm{~s}^{-1}$, respectively, and electron mobilities were measured to be $3.04 \times 10^{-4} \mathrm{~cm}^{2} \mathrm{~V}^{-1} \mathrm{~s}^{-1}$ and $2.08 \times 10^{-4}$ $\mathrm{cm}^{2} \mathrm{~V}^{-1} \mathrm{~s}^{-1}$, respectively. These results reflect that the morphology of this system is efficient for charge transport.

\section{Discussion}

In summary, we demonstrate that the two NIR-absorbing multifused benzotrizole-based NFAs, Y1 and Y2, blending with a commercialized donor PBDB-T can achieve a high PCE of $13.42 \%$ and $13.40 \%$, respectively. The high performance was enabled by the relatively high $\mathrm{EQE}_{\mathrm{EL}}$ of approximately $0.5 \times 10^{-4}$, which indicates low nonradiative recombination loss of the blend. In addition, despite small donor-acceptor energy offsets, highly efficient charge generation efficiencies have been shown for both PBDB-T:Y1 and PBDB-T:Y2 blend films. Moreover, a homogeneous and nanophase-segregated structure with an optimized size and preferred orientation are also observed in blend films, which contributed to the efficient charge separation and transport. These results are achieved using a simple design strategy by incorporation of nitrogen acting as a bridging atom between adjacent electrondonor (thieno[3,2-b]thiophene) segment and weak electrondeficient segment (benzotriazole) (DAD fused core), leading to strong absorption in Vis-NIR region. A certified high efficiency of single-junction OPVs meeting the ISO 17025 Standards was achieved at 12.6\% based on PBDB-T:Y1 devices. This work provides a rational route to a delicate design with relatively tight spacing using the benzotrizole-based DAD structure as central core towards high-performance NFAs.

\section{Methods}

UV-Vis absorption spectra and cyclic voltammetry measurement. UV-Vis absorption spectra were recorded on the SHIMADZU UV-4100 spectrophotometer. For the solid-state measurements, Y1 and Y2 solutions in chloroform were spin-coated on quartz plates. The CV results were obtained with a computercontrolled CHI 660E electrochemical workstation using polymer or NFA films on platinum electrode $\left(1.0 \mathrm{~cm}^{2}\right)$ as the working electrode, a platinum wire as the counter electrode and $\mathrm{Ag} / \mathrm{AgCl}(0.1 \mathrm{M})$ as the reference electrode in an anhydrous and argon-saturated solution of $0.1 \mathrm{M}$ of tetrabutylammonium hexafluorophosphate $\left(\mathrm{Bu}_{4} \mathrm{NPF}_{6}\right)$ in acetonitrile at a scanning rate of $50 \mathrm{mV} \mathrm{s}^{-1}$. Electrochemical onsets were determined at the position where the current started to rise from the baseline.

Photoluminescence and electroluminescence spectra. PL and EL spectra were measured using a light guide positioned close to the sample. The excitation light of PL measurement was peaked at $530 \mathrm{~nm}$ and a $650 \mathrm{~nm}$-long-pass filter was used during the measurement. The bias of EL measurement was applied on the devices using a Keithley 2400 SourceMeter. The detector was a Newton EM-CCD Si array detector at $-60^{\circ} \mathrm{C}$ with a Shamrock SR-303i spectrograph from Andor Tech. PLQY values were obtained using an integrating sphere.

\footnotetext{
Atomic force microscopy(AFM) and transmission electron microscopy(TEM). The morphologies of the polymer/acceptor blend films were investigated by AFM (Bruker Dimension Fast Scan Scanning Probe Microscope) in contact mode using a 1 and $5 \mu \mathrm{m}$ (Supplementary Fig. 17) scanners. Samples for the TEM measurements were prepared as following: The active layer films were spin-casted on ITO/poly (3,4-ethylenedioxythiophene) poly(styrenesulfonate) (PEDOT:PSS) substrates, and the substrates with active layers were submerged in deionized water to make the active layers floats onto the air-water interface. Then, the floated films were picked up on an unsupported 200 mesh copper grids for the TEM measurements. TEM measurements were conducted with a T12 cryo-electron microscope.
}

Grazing incidence wide-angle X-ray scattering measurements(GIWAXS).

GIWAXS measurement was performed at Advanced Light Source, Lawrence Berkeley National Laboratory on the 7.3.3 beamline. All the samples were deposited on the silicon wafer with $100 \mathrm{~nm}$ silicon oxide. Samples were irradiated by $10 \mathrm{keV}$ at a fixed X-ray incident angle of $0.12^{\circ}$ with an exposure time of $3 \mathrm{~s}$. All measurements were conducted under a helium atmosphere to reduce air scattering.

Fabrication and measurement of devices. The ITO glass was precleaned in an ultrasonic bath of detergent, deionized water, acetone and isopropanol, and UVtreated in ultraviolet-ozone chamber (Jelight Company, USA) for $15 \mathrm{~min}$. A thin layer $(30 \mathrm{~nm})$ of $\mathrm{ZnO}$ was spin-coated onto the ITO glass by sol-gel method and baked at $200^{\circ} \mathrm{C}$ for $60 \mathrm{~min}$. The polymer PBDB-T:Y1 or Y2

$\left(\mathrm{D}: \mathrm{A}=1: 1,16 \mathrm{mg} \mathrm{mL}^{-1}\right.$ in total) were dissolved in chlorobenzene (CB) and 1-chloronaphthalene $(\mathrm{CN})\left(0.8 \%\right.$, v/v) overnight $\left(80^{\circ} \mathrm{C}\right)$ and spin-cast at $3000 \mathrm{rpm}$ for $60 \mathrm{~s}$ onto the $\mathrm{ZnO}$ layer. The thickness of the photoactive layer is about $100 \mathrm{~nm}$ measured by Ambios Technology XP-2 profilometer. A bilayer cathode consisting of $\mathrm{MoO}_{3}(15 \mathrm{~nm})$ capped with $\mathrm{Ag}(150 \mathrm{~nm})$ was thermal evaporated under a shadow mask with a base pressure of ca. $10^{-5} \mathrm{~Pa}$. Finally, top electrodes were deposited in a vacuum onto the active layer. The active area of the device was 0.1 $\mathrm{cm}^{2}$. The certified cells have the area of $0.0548 \mathrm{~cm}^{2}$, which is defined by a metal mask with an aperture aligned with the device area.

$J-V$ characteristics of photovoltaic cells were taken using a Keithley 2400 source measure unit under a simulated AM $1.5 \mathrm{G}$ spectrum with an Oriel 9600 solar simulator. We also conducted both forward and backward scans of PBDB-T:Y1, which yielded identical results (Supplementary Fig. 13). EQEs were measured using an integrated system (Enlitech, Taiwan) and lock-in amplifiers with a current preamplifier under short-circuit conditions.

Asymptotic $P_{\max }$ protocol, which was proposed by the National Renewable Energy Laboratory's (NREL) Cell and Module Performance group and accredited to ISO 17025 Standards, can be taken as a reliable evaluation method for the thin film photovoltaics. The optimized device (PBDB-T:Y1) with the PCE of $13.13 \%$ ( $V_{\mathrm{OC}}$ of $0.87 \mathrm{~V}, J_{\mathrm{SC}}$ of $21.90 \mathrm{~mA} \mathrm{~cm}^{-2}$ and $\mathrm{FF}$ of $69.72 \%$ ) was sent to an accredited solar cell calibration laboratory (Newport Corp.) for certification. Current-voltage parameters were measured under a 13-point IV sweep configuration wherein the bias voltage (current for $V_{\mathrm{OC}}$ determination) is held constant until the measured current (voltage for $V_{\mathrm{OC}}$ ) is determined to be $0.05 \%$ level.

$\mathbf{E Q E}_{\mathrm{EL}}$ measurement. $\mathrm{EQE}_{\mathrm{EL}}$ values were obtained from an in-house-built system including a Hamamatsu silicon photodiode 1010B, a Keithley 2400 SourceMeter to provide voltage and record injected current, and a Keithley 485 Picoammeter to measure the emitted light intensity.

FTPS-EQE measurement. FTPS-EQE was measured using Vertex 70 from Bruker Optics, equipped with a quartz tungsten halogen lamp, quartz beam splitter and external detector option. A low-noise current amplifier (SR570) was used to amplify the photocurrent produced on illumination of the photovoltaic devices with light modulated by the Fourier-transform infrared spectroscope (FTIR). The output voltage of the current amplifier was fed back into the external detector port of the FTIR, to be able to use the FTIR's software to collect the photocurrent spectrum.

Optical gap calculation. We determined the optical gaps from the intersection between normalized emission and absorption spectra ${ }^{47}$.

Hole mobility and electron mobility measurements. The hole-only or electron only diodes were fabricated using the following architectures: ITO/poly(3,4-ethylenedioxythiophene):poly(styrenesulfonate) (PEDOT:PSS)/active layer/ gold (Au) for holes and ITO/ZnO/active layer/Al for electrons. Mobilities were extracted by fitting the current density-voltage curves using the Mott-Gurney relationship (SCLC). The mobilities were obtained by taking current-voltage curves and fitting the results to a space charge limited form.

Time-resolved photoluminescence measurement. The time-resolved PL measurements were performed at room temperature. A wavelength-tunable modelocked Ti:Sapphire laser was used as the excitation source, which has a repetition rate of $76 \mathrm{MHz}$ and a pulse duration of $2 \mathrm{ps}$. Here, the laser wavelength was set at $725 \mathrm{~nm}$. PL emission induced by the pulsed excitation laser was detected by a Hamamatsu streak camera combined with a single-grating monochromator.

\section{Data availability}

The data that support the findings of this study are available from the corresponding author upon reasonable request. 
Received: 10 September 2018 Accepted: 7 January 2019

Published online: 04 February 2019

\section{References}

1. Yu, G., Gao, J., Hummelen, J. C., Wudl, F. \& Heeger, A. J. Polymer photovoltaic cells: enhanced efficiencies via a network of internal donoracceptor heterojunctions. Science 270, 1789-1791 (1995).

2. Halls, J. J. M., Pichler, K., Friend, R. H., Moratti, S. C. \& Holmes, A. B. Exciton diffusion and dissociation in a poly( $p$ - phenylenevinylene)/C60 heterojunction photovoltaic cell. Appl. Phys. Lett. 68, 3120-3122 (1996).

3. Li, G., Zhu, R. \& Yang, Y. Polymer solar cells. Nat. Photon 6, 153-161 (2012).

4. Lu, L. et al. Recent advances in bulk heterojunction polymer solar cells. Chem. Rev. 115, 12666-12731 (2015).

5. Vohra, V. et al. Efficient inverted polymer solar cells employing favourable molecular orientation. Nat. Photon. 9, 403-408 (2015).

6. Li, Y. Molecular design of photovoltaic materials for polymer solar cells: toward suitable electronic energy levels and broad absorption. Acc. Chem. Res. 45, 723-733 (2012).

7. Kaltenbrunner, M. et al. Ultrathin and lightweight organic solar cells with high flexibility. Nat. Commun. 3, 770 (2012).

8. Li, G. et al. High-efficiency solution processable polymer photovoltaic cells by self-organization of polymer blends. Nat. Mater. 4, 864-868 (2005)

9. Brabec, C. J., Heeney, M., McCulloch, I. \& Nelson, J. Influence of blen microstructure on bulk heterojunction organic photovoltaic performance. Chem. Soc. Rev. 40, 1185-1199 (2011).

10. Peet, J., Heeger, A. J. \& Bazan, G. C. "Plastic" solar cells: self-assembly of bulk heterojunction nanomaterials by spontaneous phase separation. Acc. Chem. Res. 42, 1700-1708 (2009).

11. Svensson, M. et al. High performance polymer solar cells of an alternating polyfluorene copolymer and a fullerene derivative. Adv. Mater. 15, 988-991 (2003).

12. Liu, J. et al. Fast charge separation in a non-fullerene organic solar cell with a small driving force. Nat. Energy 1, 16089 (2016).

13. Yao, J. et al. Quantifying losses in open-circuit voltage in solution-processable solar cells. Phys. Rev. Appl. 4, 014020 (2015).

14. Dang, M. T., Hirsch, L. \& Wantz, G. P3HT:PCBM, best seller in polymer photovoltaic research. Adv. Mater. 23, 3597-3602 (2011).

15. Linderl, T. et al. Energy losses in small-molecule organic photovoltaics. $A d v$. Energy Mater. 7, 1700237 (2017).

16. Benduhn, J. et al. Intrinsic non-radiative voltage losses in fullerene-based organic solar cells. Nat. Energy 2, 17053 (2017).

17. Vandewal, K., Tvingstedt, K., Gadisa, A., Inganäs, O. \& Manca, J. V. Relating the open-circuit voltage to interface molecular properties of donor:acceptor bulk heterojunction solar cells. Phys. Rev. B 81, 125204 (2010).

18. Würfel, P. \& Würfel, U. Physics of Solar Cells: From Basic Principles to Advanced Concepts (John Wiley \& Sons, New York, 2009).

19. Zhang, G. et al. Nonfullerene acceptor molecules for bulk heterojunction organic solar cells. Chem. Rev. 118, 3447-3507 (2018).

20. Vandewal, K. et al. Efficient charge generation by relaxed charge-transfer states at organic interfaces. Nat. Mater. 13, 63-68 (2014).

21. Vandewal, K., Tvingstedt, K., Gadisa, A., Inganäs, O. \& Manca, J. V. On the origin of the open-circuit voltage of polymer-fullerene solar cells. Nat. Mater. 8, 904-909 (2009).

22. Burke, T. M., Sweetnam, S., Vandewal, K. \& McGehee, M. D. Beyond Langevin recombination: how equilibrium between free carriers and charge transfer states determines the open-circuit voltage of organic solar cells. Adv. Energy Mater. 5, 1500123 (2015)

23. Qian, D. et al. Design rules for minimizing voltage losses in high-efficiency organic solar cells. Nat. Mater. 17, 703 (2018).

24. Yan, C. et al. Non-fullerene acceptors for organic solar cells. Nat. Rev. Mater. 3, 18003 (2018).

25. Cheng, P., Li, G., Zhan, X. \& Yang, Y. Next-generation organic photovoltaics based on non-fullerene acceptors. Nat. Photon 12, 131-142 (2018).

26. Fei, Z. et al. An alkylated indacenodithieno[3,2-b]thiophene-based nonfullerene acceptor with high crystallinity exhibiting single junction solar cell efficiencies greater than $13 \%$ with low voltage losses. Adv. Mater. 30, 1705209 (2018).

27. Jiang, W. et al. Ternary nonfullerene polymer solar cells with $12.16 \%$ efficiency by introducing one acceptor with cascading energy level and complementary absorption. Adv. Mater. 30, 1703005 (2017).

28. Li, T. et al. Fused Tris(thienothiophene)-based electron acceptor with strong near-infrared absorption for high-performance As-cast solar cells. Adv. Mater. 30, 1705969 (2018).
29. Kaya, E., Balan, A., Baran, D., Cirpan, A. \& Toppare, L. Electrochromic and optical studies of solution processable benzotriazole and fluorene containing copolymers. Org. Electron. 12, 202-209 (2011).

30. Chen, X., Jin, Q., Wu, L., Tung, C. \& Tang, X. Synthesis and unique photoluminescence properties of nitrogen-rich quantum dots and their applications. Angew. Chem. Int. Ed. 53, 12542-12547 (2014)

31. Banal, J. L. et al. Electron deficient conjugated polymers based on benzotriazole. Polym. Chem. 4, 1077-1083 (2013).

32. Ghosh, S., Bedi, A. \& Zade, S. S. Thienopyrrole and selenophenopyrrole donor fused with benzotriazole acceptor: microwave assisted synthesis and electrochemical polymerization. RSC Adv. 5, 5312-5320 (2015).

33. Li, P., Wang, Z., Song, C. \& Zhang, H. Rigid fused $\pi$-spacers in D- $\pi-A$ type molecules for dye-sensitized solar cells: a computational investigation. J. Mater. Chem. C 5, 11454-11465 (2017).

34. Hosseinzadeh, E. \& Hadipour, N. L. The influence of the structural variations of the fused electron rich-electron deficient unit in the $\pi$-spacer of A-D- $\pi$-D-A organic dyes on the efficiency of dye-sensitized solar cells: A computational study. Org. Electron. 62, 43-55 (2018).

35. Zhao, W. et al. Fullerene-free polymer solar cells with over $11 \%$ efficiency and excellent thermal stability. Adv. Mater. 28, 4734-4739 (2016).

36. Cheng, Y. J. et al. Thieno[3,2-b]pyrrolo donor fused with benzothiadiazolo, benzoselenadiazolo and quinoxalino acceptors: synthesis, characterization, and molecular properties. Org. Lett. 13, 5484-5487 (2011)

37. Lin, Y. et al. An electron acceptor challenging fullerenes for efficient polymer solar cells. Adv. Mater. 27, 1170-1174 (2015)

38. Zhang, Y. et al. Conjugated polymers based on $\mathrm{C}, \mathrm{Si}$ and N-bridged dithiophene and thienopyrroledione units: synthesis, field-effect transistors and bulk heterojunction polymer solar cells. J. Mater. Chem. 21, 3895 (2011)

39. Feng, L. et al. Thieno[3,2-b]pyrrolo-fused pentacyclic benzotriazole-based acceptor for efficient organic photovoltaics. Acs Appl. Mater. Interfaces $\mathbf{9}$, 31985-31992 (2017)

40. Hou, J., Inganas, O., Friend, R. H. \& Gao, F. Organic solar cells based on nonfullerene acceptors. Nat. Mater. 17, 119-128 (2018).

41. Liu, X. et al. Efficient organic solar cells with extremely high open-circuit voltages and low voltage losses by suppressing nonradiative recombination losses. Adv. Energy Mater. 8, 1801699 (2018).

42. Nikolis, V. C. et al. Reducing voltage losses in cascade organic solar cells while maintaining high external quantum efficiencies. Adv. Energy Mater. 7, 1700855 (2017).

43. Clarke, T. M. \& Durrant, J. R. Charge photogeneration in organic solar cells. Chem. Rev. 110, 6736-6767 (2010).

44. Shockley, W. \& Queisser, H. J. Detailed balance limit of efficiency of p-n junction solar cells. J. Appl. Phys. 32, 510-519 (1961).

45. Rivnay, J., Mannsfeld, S. C., Miller, C. E., Salleo, A. \& Toney, M. F. Quantitative determination of organic semiconductor microstructure from the molecular to device scale. Chem. Rev. 112, 5488-5519 (2012).

46. Malliaras, G., Salem, J., Brock, P. \& Scott, C. Electrical characteristics and efficiency of single-layer organic light-emitting diodes. Phys. Rev. B 58, R13411 (1998).

47. Wang, Y. et al. Optical gaps of organic solar cells as a reference for comparing voltage losses. Adv. Energy Mater. 8, 1901352 (2018).

48. National Renewable Energy Laboratory: Best Research-Cell Efficiencies chart. https://www.nrel.gov/pv/assets/pdfs/pv-efficiency-chart.20190103.pdf (2018) Accessed 23 January 2018

\section{Acknowledgements}

Y.Y. acknowledges the Air Force Office of Scientific Research (AFOSR) (FA2386-15-14108, FA9550e15-1e0610, and FA9550-15-1-0333) and UC-Solar Program (MRPI 328368) for their financial support. Part of this research was performed in beamline 7.3.3 in Advanced Light Source Lawrence Berkeley National Laboratory. Y.Z. acknowledges the National Key Research \& Development Projects of China (2017YFA0206600), National Natural Science Foundation of China (21875286), Science Fund for Distinguished Young Scholars of Hunan Province (2017JJ1029). F.G. acknowledges the Swedish Energy Agency Energimyndigheten (2016-010174) and the Swedish Government Strategic Research Area in Materials Science on Functional Materials at Linköping University (Faculty Grant No. SFO-Mat-LiU \#2009-00971). The authors also thank Dr. Rui Zhu for acquiring GIWAXS experiment, Mrs. Mei Luo for synthesizing raw materials, Mr. Can Zhu for fabricating the solar cell devices, Dr. Jin-Wook Lee for thermogravimetric analysis experiments, Dr. Galia Pozina for help with the PL decay measurements, and Ms. Selbi Nuryyeva and Mr. Beibei Qiu for providing important insights on the manuscript.

\section{Author contributions}

Y.Y., Y.Z. and J.Y. conceived and directed the experiments. J.Y. and Z.Z. synthesized the non-fullerene acceptors and carried out the NMR and high-resolution mass spectrum measurements, ultraviolet and cyclic voltammetry; T.H. and P.C. fabricated the solar cell 
devices; H.Z. performed the FTPS, EL and PL experiment supervised by F.G.; S.-Y.C. carried out the atomic force microscopy and transmission electron microscopy. W.H. and R.W. analyzed the GIWAXS data. D.M. helped in the data interpretation. J.Y. made the first draft; all authors contributed to analysis and writing.

\section{Additional information}

Supplementary Information accompanies this paper at https://doi.org/10.1038/s41467019-08386-9.

Competing interests: The authors declare no competing interests.

Reprints and permission information is available online at http://npg.nature.com/ reprintsandpermissions/

Journal peer review information: Nature Communications thanks the anonymous reviewers for their contribution to the peer review of this work.
Publisher's note: Springer Nature remains neutral with regard to jurisdictional claims in published maps and institutional affiliations.

(c) (i) Open Access This article is licensed under a Creative Commons Attribution 4.0 International License, which permits use, sharing, adaptation, distribution and reproduction in any medium or format, as long as you give appropriate credit to the original author(s) and the source, provide a link to the Creative Commons license, and indicate if changes were made. The images or other third party material in this article are included in the article's Creative Commons license, unless indicated otherwise in a credit line to the material. If material is not included in the article's Creative Commons license and your intended use is not permitted by statutory regulation or exceeds the permitted use, you will need to obtain permission directly from the copyright holder. To view a copy of this license, visit http://creativecommons.org/ licenses/by/4.0/.

(C) The Author(s) 2019 